\title{
OBTENCIÓN DE PARTÍCULAS DE TiO, POR SOL-GEL, ASISTIDO CON ULTRASONIDO PARA APLICACIONES FOTOCATALÍTICAS
}

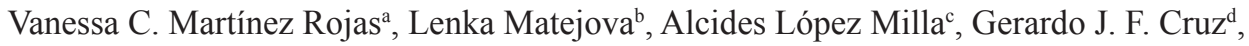 \\ José L. Solís Veliza, Mónica M. Gómez León ${ }^{* a}$
}

\begin{abstract}
RESUMEN
La síntesis de las partículas de $\mathrm{TiO}_{2}$ se realizó mediante la técnica sol-gel asistida con radiación ultrasónica a partir de una solución alcohólica de isopropóxido de titanio. Luego se sometió a un tratamiento térmico en atmósfera de aire a $350{ }^{\circ} \mathrm{C}$ por $1 \mathrm{~h}$. Difracción de rayos $\mathrm{X}$ y microscopía electrónica de transmisión confirmaron que el tamaño de los dominios cristalinos se encuentran entre 10 y $37 \mathrm{~nm}$. El estudio de espectroscopía infrarroja confirma la presencia de grupos - $\mathrm{OH}$ en la superficie del $\mathrm{TiO}_{2}$. La modificación de la morfología y el área superficial, debido a la influencia del tiempo de exposición a la radiación ultrasónica, se evidencia con los estudios de microscopía electrónica de barrido y BET, respectivamente. Las propiedades del $\mathrm{TiO}_{2}$ obtenido se estudiaron monitoreando la degradación de soluciones de anaranjado de metilo en presencia de radiación UV-A. Se observó que a mayor exposición de radiación ultrasónica durante la síntesis de las muestras de $\mathrm{TiO}_{2}$, mayor fue la constante de velocidad para la reacción de fotocatálisis del anaranjado de metilo.
\end{abstract}

Palabras clave: dióxido de titanio, ultrasonido, fotocatálisis, anaranjado de metilo.

\section{PRODUCTION OF TiO, PARTICLES BY SOL-GEL ULTRASOUND ASSISTED FOR PHOTOCATALYTIC APPLICATIONS}

\begin{abstract}
Synthesis of $\mathrm{TiO}_{2}$ particles was made by sol-gel technique assisted of ultrasonic radiation from an alcoholic solution of titanium isopropoxide. Then was subjected to a heat treatment in air at $350{ }^{\circ} \mathrm{C}$ for $1 \mathrm{~h}$. X-ray diffraction and transmission electron microscopy confirmed that the size of the crystalline domains is between 10 and $37 \mathrm{~nm}$. Infrared spectroscopy study confirms the presence of $-\mathrm{OH}$ groups on the surface of $\mathrm{TiO}_{2}$. Modification of the morphology and surface area, due to the influence of exposure time to the ultrasonic radiation, is evidenced by studies of SEM and BET respectively. Properties of $\mathrm{TiO} 2$ obtained were studied by monitoring the degradation of solutions of methyl orange in the presence of UV-A radiation. It was observed that larger the ultrasonic radiation exposure during the $\mathrm{TiO}_{2}$ synthesis larger the constant velocity for the photocatalytic reaction for the methyl orange.
\end{abstract}

Keywords: titanium dioxide, ultrasound, photocatalysis, methyl orange.

\footnotetext{
${ }^{\text {a }}$ Facultad de Ciencias, Universidad Nacional de ingeniería, Av. Túpac Amaru 210, Lima25, Perú. mgomez@uni.edu.pe

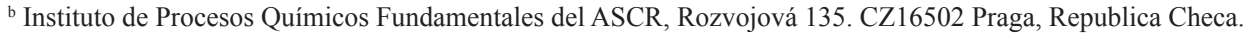

${ }^{c}$ Instituto Peruano de Energía Nuclear. Av. Canadá 1470, Lima 41, Perú.

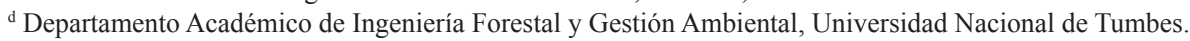




\section{INTRODUCCIÓN}

Actualmente el $\mathrm{TiO}_{2}$ es un material semiconductor de gran importancia en las aplicaciones fotocatalíticas, ${ }^{1-3}$ esto incluye la destrucción fotocatalítica de contaminantes orgánicos en aguas usadas, siendo bastante usado en temas de remediación ambiental ${ }^{4-6}$ y fotogeneración de electricidad usando celdas solares sensibilizadas con pigmentos. ${ }^{7-10}$

El dióxido de titanio $\left(\mathrm{TiO}_{2}\right)$ se encuentra en la naturaleza en forma de tres estructuras cristalinas: rutilo, anatasa y broquita. La anatasa y el rutilo son los más comunes; ambos son de estructuras tetragonales y con similares densidades. No tienen absorción en la región visible ni en el IR cercano y, en forma monocristalina, tienen energías de banda prohibida de $3,0 \mathrm{eV}$ (rutilo) y $3,2 \mathrm{eV}$ (anatasa). ${ }^{11}$ Siendo la fase anatasa la que presenta mejor actividad fotocatalítica. ${ }^{11-13}$

Entre las propiedades más importantes del $\mathrm{TiO}_{2}$ están su buena dispersabilidad en soluciones orgánicas y su muy baja citotoxicidad, ${ }^{14,15}$ razones por las que es ampliamente usado en protectores solares ${ }^{16}$, pastas dentífricas, ${ }^{17}$ polvos cosméticos ${ }^{18}$ y pigmentos. ${ }^{19}$

\section{PARTE EXPERIMENTAL}

Los polvos de $\mathrm{TiO}_{2}$ se sintetizaron por la ruta sol-gel modificada con ultrasonido, empleando un baño ultrasónico Cole-Parmer 8891 operado a $42 \mathrm{kHz}$ y 130W. Se preparó una solución al 25\% V/V de isopropóxido de titanio (Merck, 99,9\%) en etanol (Merck, 99,9\%).

En la figura 1 se muestra el esquema del proceso, en el matraz se encuentra la solución alcohólica de isopropóxido de titanio irradiándose en el baño ultrasónico, teniendo cuidado de ubicar el matraz a la altura adecuada, de modo que se observe la mayor cantidad de burbujas verificando que se encuentre muy bien tapado el matraz para evitar la evaporación de los reactivos.

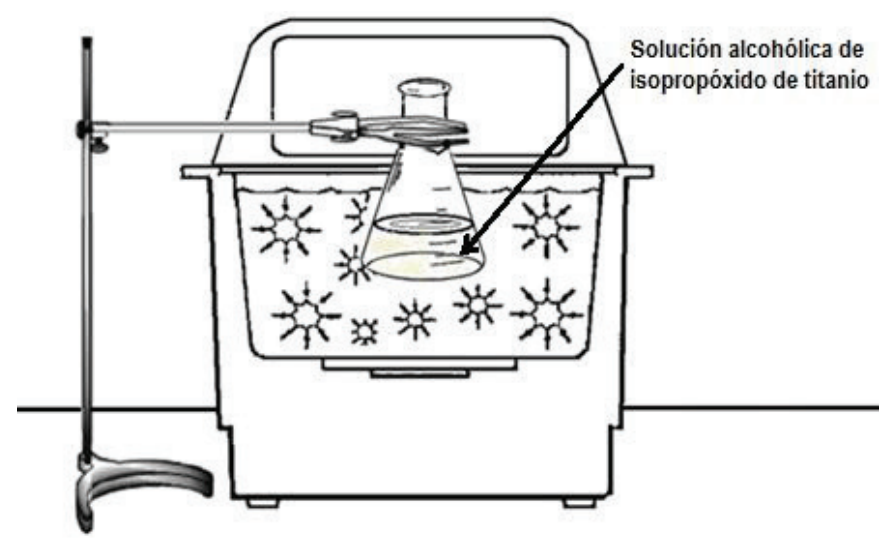

Figura 1. Montaje experimental empleado en la síntesis sol gel de $\mathrm{TiO}_{2}$ asistida con ultrasonido. ${ }^{20}$ 
El material sólido obtenido se lavó con agua destilada, se centrifugó y se secó a $80{ }^{\circ} \mathrm{C}$ durante dos horas.

Las reacciones propuestas en la síntesis de $\mathrm{TiO}_{2}$ por el proceso sol-gel asistido con radiación ultrasónica, según lo propuesto por otros autores podrían ser:

$$
\begin{aligned}
& \mathrm{Ti}(\mathrm{OR})_{4}+4 \mathrm{H}_{2} \mathrm{O} \rightarrow 2 \mathrm{Ti}(\mathrm{OH})_{4}+4 \mathrm{ROH} \\
& \mathrm{Ti}(\mathrm{OH})_{4} \rightarrow \mathrm{TiO}_{2} \cdot \mathrm{XH}_{2} \mathrm{O}+(2-\mathrm{X}) \mathrm{H}_{2} \mathrm{O}
\end{aligned}
$$

donde $\mathrm{R}$ es el grupo etil y X puede tomar el valor de 1 o $2 .{ }^{21}$

La obtención del $\mathrm{TiO}_{2}$ se realizó modificando el tiempo de irradiación ultrasónica tru; se trabajó para 1,2 y $3 \mathrm{~h}$.

La estructura y el tamaño de dominio cristalino del $\mathrm{TiO}_{2}$ se determinó por difracción de rayos $\mathrm{X}$ empleando un difractómetro Rigaku Miniflez II Desktop operado con radiación $\mathrm{CuK} \alpha(0,15045 \mathrm{~nm})$ a $30 \mathrm{kV}$ y $20 \mathrm{~mA}$ con una velocidad de $3 \% \mathrm{~min}$. La microestructura del $\mathrm{TiO}_{2}$ también fue analizada por microscopía electrónica de transmisión (MET) usando un EFTEM LEO 912 OMEGA, y microscopía electrónica de barrido (MEB) empleando el microscopio Tescan Vega 3. Esta información fue complementada con un estudio del material por espectroscopía infrarroja con transformada de Fourier empleando un equipo Shimadzu, modelo FTIR-8300. El material sintetizado fue aplicado para evaluaciones fotocatalíticas, en las que el área superficial es un factor importante; por ello se realizó el estudio del área superficial total de acuerdo a la Teoría de Brunauer-Emmett-Teller (BET) ${ }^{22}$ empleando el equipo Micromeritics Gemini VII serie T.

La evaluación de las propiedades fotocatalíticas se estudiaron en un sistema como el mostrado en la figura 2, que consiste de una fuente de radiación UV, Ultra-Vitalux OSRAM (220 V, $300 \mathrm{~W}$ ), un vaso que contiene la solución a degradar con el $\mathrm{TiO}_{2}$ en suspensión y constante agitación. Para mantener la temperatura de la solución se empleó un baño de agua. Todo el sistema se operó dentro de una cámara de paredes negras.

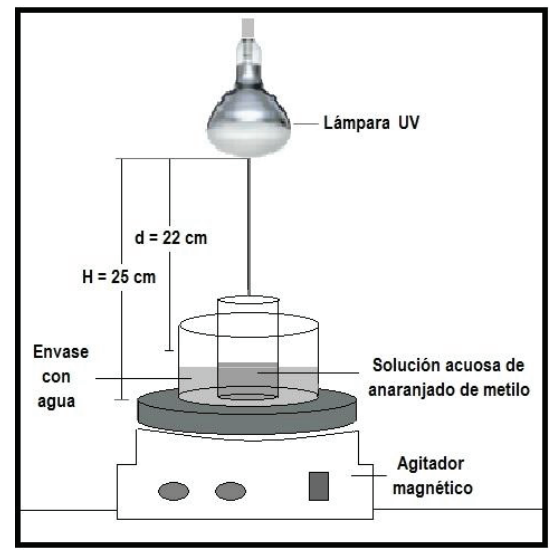

Figura 2. Esquema del fotorreactor empleado para la degradación del anaranjado de metilo. 
Las evaluaciones de fotodegradación se estudiaron en soluciones de anaranjado de metilo de concentración inicial igual a $6,11 \times 10^{-5} \mathrm{~mol} / \mathrm{L}$.

\section{RESULTADOS Y DISCUSIÓN}

Las partículas de $\mathrm{TiO}_{2}$ recién sintetizadas no presentaron estructura cristalina por lo que se aplicó tratamiento térmico a $350{ }^{\circ} \mathrm{C}$ durante $1 \mathrm{~h}$, obteniéndose para todos los casos la fase anatasa. La figura 3 muestra el difractograma de rayos $\mathrm{X}$ hecho a una muestra sonicada durante $3 \mathrm{~h}$, sin tratamiento térmico y con tratamiento térmico de $350{ }^{\circ} \mathrm{C}$ durante $1 \mathrm{~h}$.

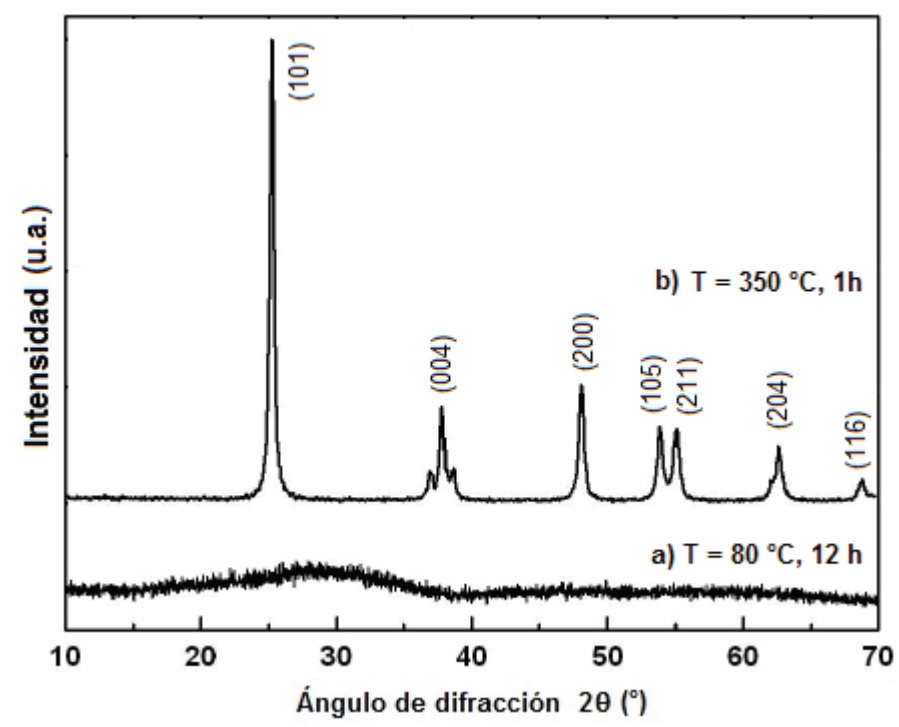

Figura 3. Difractogramas de rayos $\mathrm{X}$ de las partículas de $\mathrm{TiO}_{2}$ (a) recién sintetizadas y b) luego del tratamiento térmico a $350{ }^{\circ} \mathrm{C}$ durante $1 \mathrm{~h}$.

El estudio estructural de las muestras obtenidas según su dependencia de tru, se realizó tratándolas térmicamente a $350{ }^{\circ} \mathrm{C}$ por una hora.

La figura 4 muestra los difractogramas de estas partículas. Se puede observar que todas poseen la fase anatasa. Además, se observa el engrosamiento de los picos con el aumento de tru. 


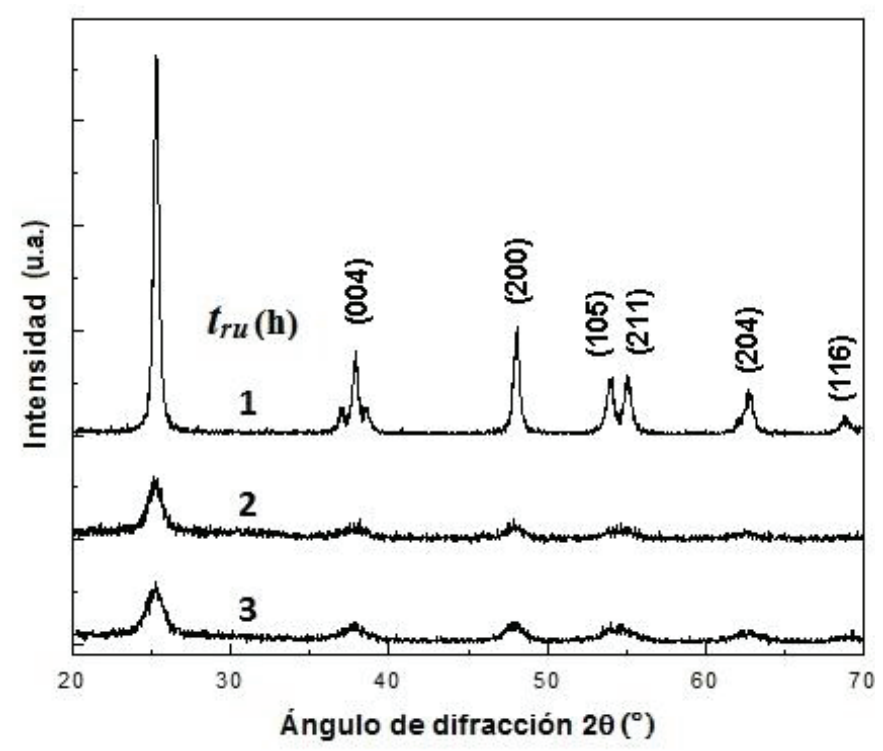

Figura 4. Difractogramas de las partículas de $\mathrm{TiO}_{2}$ obtenidas con diferentes tiempos de radiación ultrasónica, denotado tru, durante la síntesis.

El tamaño del dominio cristalino medio, calculado empleando la fórmula de Debye Scherrer y con el programa TOPAS, fue de 37, 22 y $10 \mathrm{~nm}$ para las muestras 1,2 y 3, respectivamente. Se observó una disminución sostenida del dominio cristalino a medida que la síntesis se realiza con un mayor tiempo de irradiación, tru.

La información estructural y morfológica del $\mathrm{TiO}_{2}$ fue también estudiada empleando MET. La figura 5 muestra la micrografía electrónica de transmisión de las partículas de $\mathrm{TiO}_{2}$ sintetizado a $3 \mathrm{~h}$ de ultrasonido, con la inserción del patrón de difracción de electrones que corresponde a la fase anatasa del $\mathrm{TiO}_{2}$. El tamaño aproximado del dominio cristalino es 10 $\mathrm{nm}$, valor que presenta muy buena coincidencia con la información obtenida por difracción de rayos $X$.

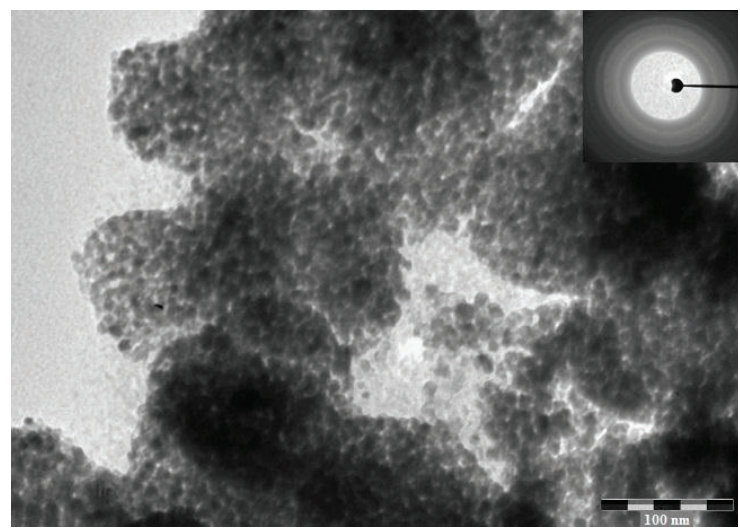

Figura 5. Micrografía y patrón de difracción de electrones de nanopartículas de $\mathrm{TiO}_{2}$ sintetizadas con tru igual a $3 \mathrm{~h}$ y sometidas a un tratamiento térmico de $350{ }^{\circ} \mathrm{C}$ durante $1 \mathrm{~h}$. 
El estudio de la morfología de las partículas de $\mathrm{TiO}_{2}$ realizado por MEB muestra claramente la influencia de la radiación ultrasónica en la síntesis del material. La figura 6 muestra las micrografías de las tres condiciones de síntesis empleadas.
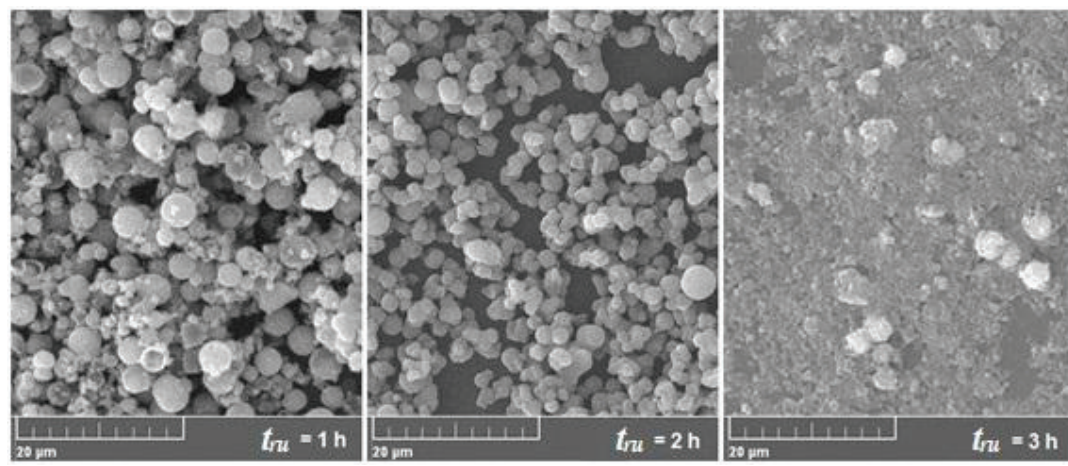

Figura 6. Micrografías electrónicas de barrido de los polvos de $\mathrm{TiO}_{2}$ obtenidos con diferentes tiempos de irradiación de ultrasonido tru.

Las partículas de los grupos 1 y 2 presentan morfología esférica, teniendo un diámetro promedio entre 4 y $1 \mu \mathrm{m}$; se observa que el tamaño de las aglomeraciones disminuye a medida que se aumenta el tiempo de irradiación de ultrasonido. Así, el tamaño de las partículas del grupo 3 es menor que $1 \mu \mathrm{m}$.

La información estructural del $\mathrm{TiO}_{2}$ obtenido, también fue analizado por espectroscopía infrarroja con transformada de Fourier. La figura 7 muestra el espectro FTIR, para una muestra de $\mathrm{TiO}_{2}$ sonicada a $3 \mathrm{~h}$. Se observa un pico entre 400 y $800 \mathrm{~cm}^{-1}$ debido al $\mathrm{TiO}_{2}$ asignado al stretching del enlace O-Ti-O. En el rango de $3400 \mathrm{~cm}^{-1}$ a $3600 \mathrm{~cm}^{-1}$ el pico formado se puede atribuir a la absorción de $\mathrm{OH}$, que podría provenir de moléculas de agua adsorbidas del medio circundante. Por otro lado, el pico en $1630 \mathrm{~cm}^{-1}$ es asignado al stretching de carboxilatos de titanio, formados a partir del isopropóxido de titanio y etanol como precursores.

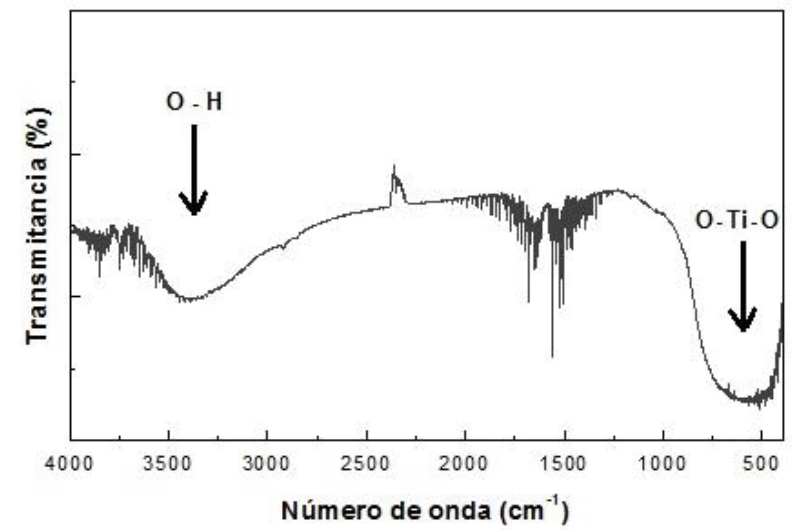

Figura 7. Espectro FTIR de las nanopartículas de $\mathrm{TiO}_{2}$ expuestas a 3 horas de radiación ultrasónica. 
Los análisis del área superficial total, así como el tamaño de poro de las muestras de $\mathrm{TiO}_{2}$ obtenidas por tiempos de sonicación entre 1 y $3 \mathrm{~h}$ se observan en la tabla 1 . De acuerdo al perfil de las isotermas, todas corresponden a un sólido mesoporoso, es decir, el diámetro de los poros está entre 2 y $50 \mathrm{~nm}^{23}$ (ver figura 8).

Tabla 1. Valores de área superficial y tamaño de poro.

\begin{tabular}{ccc}
\hline$t_{r u}(\mathrm{~h})$ & BET área superficial $\left(\mathrm{m}^{2} / \mathrm{g}\right)$ & $\begin{array}{c}\text { Tamaño de } \\
\text { poro }(\mathrm{nm})\end{array}$ \\
1 & 158,1 & 7,5 \\
2 & 193,3 & 4,5 \\
3 & 163,2 & 6,0 \\
\hline
\end{tabular}
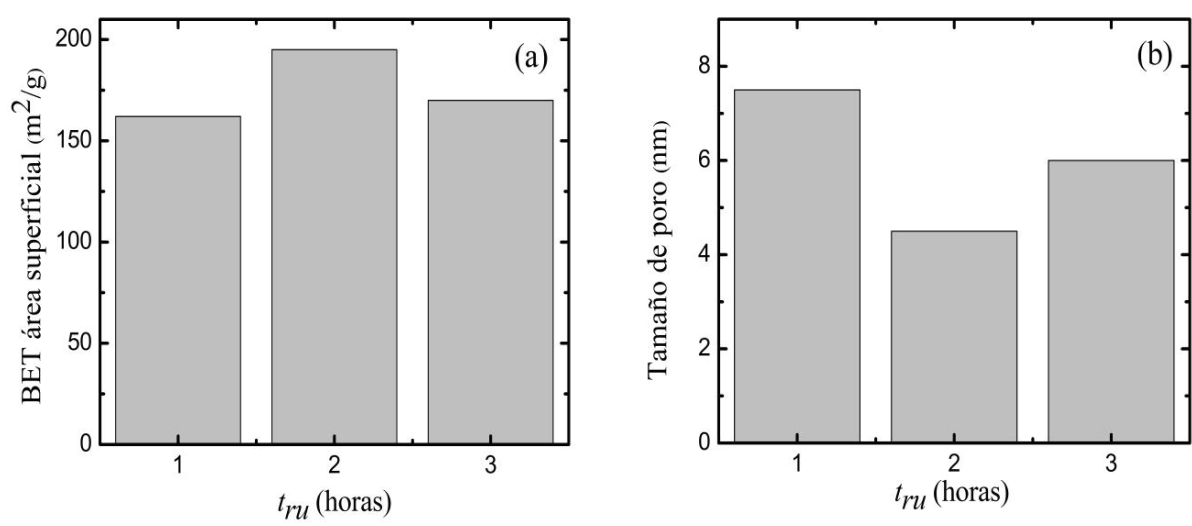

Figura 8. Histogramas de (a) área superficial promedio y (b) tamaño de poro para los diferentes tru.

El estudio de las propiedades fotocatalíticas de las partículas de $\mathrm{TiO}_{2}$ con el colorante anaranjado de metilo se muestra en la figura 9, donde se describe la evolución de la concentración del colorante en función del tiempo para tres muestras de $\mathrm{TiO}_{2}$ sintetizadas para diferentes tru. La concentración inicial del colorante para todas las evaluaciones fue de $6,11 \times 10^{-5} \mathrm{~mol} /$ litro y las medidas de su concentración se realizaron cada $30 \mathrm{~min}$. La concentración del colorante se realizó colorimétricamente empleando un espectrofotómetro Lamda 25 usado en el rango de longitud de onda de 300 a $800 \mathrm{~nm}$. 


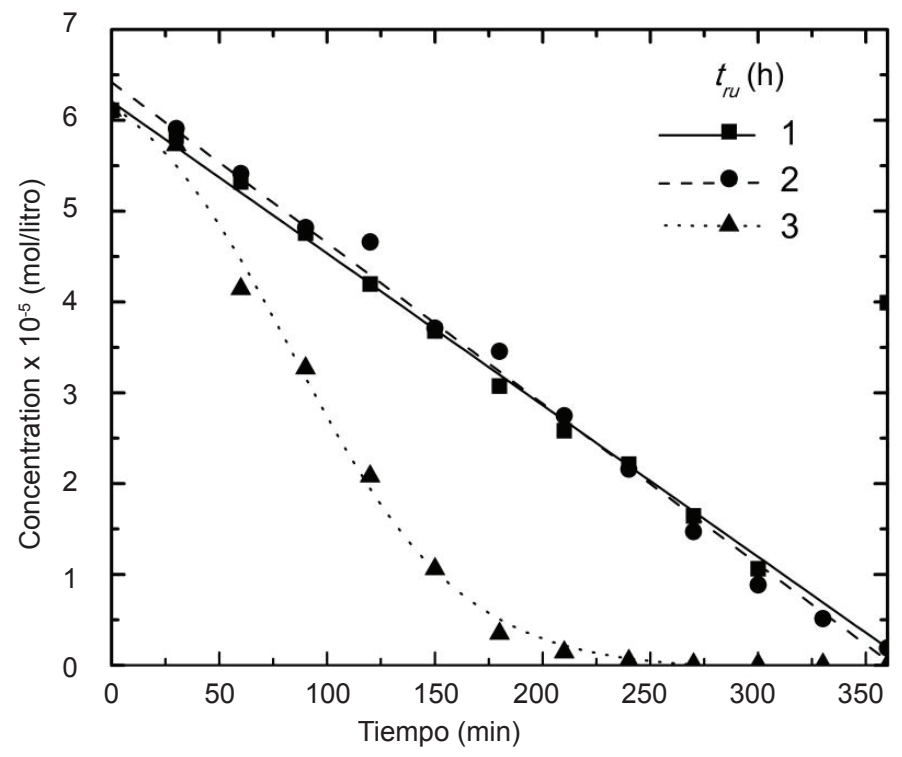

Figura 9. Evolución de la concentración de anaranjado de metilo degradado fotocatalíticamente con muestras de partículas de $\mathrm{TiO}_{2}$ sintetizadas a los valores tru indicados.

Se determinó la eficiencia de degradación del colorante anaranjado de metilo, expresado en $\%$, hasta un tiempo de 360 min de reacción. Empleando el Modelo de Langmuir-Hinshelwood en su forma integrada, ${ }^{24}$ que considera una reacción de pseudo primer orden para la que se determinó la pendiente de la curva $\operatorname{Ln}\left(C / C_{0}\right)$ vs $t$, donde $C$ es la concentración de anaranjado de metilo en el tiempo $t$ (mol/litro), $\mathrm{C}_{0}$ es la concentración inicial de anaranjado de metilo (mol/litro) y t es el tiempo ( $\mathrm{min}$ ). Este valor de la pendiente determina la constante de velocidad aparente $(k)$. Los valores de eficiencia de degradación del colorante y la constante de velocidad aparente se muestran en la tabla 2.

Tabla 2. Valores de eficiencia de remoción y constantes de velocidad aparente.

\begin{tabular}{cccc}
\hline $\begin{array}{c}\text { Muestras de } \mathrm{TiO}_{2} \\
\text { sintetizadas para diferentes }\end{array}$ & $\begin{array}{c}\text { Eficiencia de degradación del } \\
\text { colorante después de } 360 \mathrm{~min}\end{array}$ & $k\left(\mathrm{~min}^{-1}\right)$ & $R^{2}$ \\
$t_{r u}$ & $(\%)$ & & \\
$1 \mathrm{~h}$ & 93.47 & 0,007 & 0,906 \\
$2 \mathrm{~h}$ & 96.87 & 0,0084 & 0,842 \\
$3 \mathrm{~h}$ & 98.83 & 0,0177 & 0,816 \\
\hline
\end{tabular}


Se observa que estos dos parámetros se encuentran en una relación directamente proporcional con el tiempo de sonicación durante la síntesis de las partículas de $\mathrm{TiO}_{2}$. Estos valores están en concordancia con el tamaño de partícula del $\mathrm{TiO}_{2}$ obtenido a las mismas condiciones de síntesis.

\section{CONCLUSIONES}

Se sintetizó partículas de $\mathrm{TiO}_{2}$ mediante el método sol-gel asistidas con radiación ultrasónica y finalmente fueron evaluadas para pruebas de fotocatálisis. Estas partículas se dividieron en tres grupos, donde el parámetro que varió para cada grupo fue el tiempo de irradiación ultrasónica que se aplicó. El tamaño de los dominios cristalinos determinado por DRX fue entre 10 y $37 \mathrm{~nm}$. Los resultados de MEB indican que las partículas se encuentran aglomeradas en forma de esferas de tamaño micrométrico, pero a mayor tiempo de sonicación estas aglomeraciones se van disgregando. Los resultados de MET muestran que las partículas con tiempo de sonicación de $3 \mathrm{~h}$ aún mantienen su forma esférica $\mathrm{y}$, además, verifican que el tamaño del dominio cristalino coincide con el obtenido por DRX. Los resultados de FTIR verifican la estructura del $\mathrm{TiO}_{2}$. Mientras que los resultados de BET indican una relación inversa entre el área específica superficial y el tamaño de poro de las muestras. Además a mayor tiempo de sonicación, durante la síntesis de las partículas de $\mathrm{TiO}_{2}$, se obtienen menores tamaños de éstas; lo que mejora la captación de luz UV y aumenta la eficiencia de degradación del colorante anaranjado de metilo.

\section{AGRADECIMIENTOS}

Uno de nosotros (V.M.) agradece al Instituto General de Investigación de la Universidad Nacional de Ingeniería (IGI-UNI) por la beca otorgada.

El presente trabajo fue desarrollado bajo el auspicio del Consejo Nacional de Ciencia, Tecnología e Innovación Tecnológica del Perú (CONCYTEC), Proyecto $N^{\circ}$ 002/PE/2012) y la financiación del proyecto AdMatU recibida de la Academia de Finlandia.

\section{BIBLIOGRAFÍA}

1. Fujishima A, Honda $\mathrm{K}$. $\mathrm{TiO}_{2}$ photoelectrochemistry and photocatalysis. Nature. 1972;238(5358):37-8.

2. Pinho LX, Azevedo J, Brito Â, Santos A, Tamagnini P, Vilar VJP, et al. Effect of TiO2 Photocatalysis on the Destruction of Microcystis aeruginosa Cells and Degradation of Cyanotoxins Microcystin-LR and Cylindrospermopsin. Chemical Engineering Journal. 2015; (268); 144-152.

3. García-Fernández I, Fernández-Calderero I, Polo-López MI, Fernández-Ibáñez P. Disinfection of urban effluents using solar TiO2 photocatalysis: A study of significance of dissolved oxygen, temperature, type of microorganism and water matrix. Catalysis Today. 2015;240, Part A(0):30-8.

4. Palmisano G, Augugliaro V, Pagliaro M, Palmisano L. Photocatalysis: a promising route 
for 21 st century organic chemistry. Chemical Communications. 2007; (33):3425-37.

5. Antonello A, Soliveri G, Meroni D, Cappelletti G, Ardizzone S. Photocatalytic remediation of indoor pollution by transparent TiO2 films. Catalysis Today. 2014;230:3540.

6. Choi SK, Yang HS, Kim JH, Park H. Organic dye-sensitized TiO2 as a versatile photocatalyst for solar hydrogen and environmental remediation. Applied Catalysis B: Environmental. 2012;121-122:206-13.

7. Gómez M. "Photoelectrochemical and physical properties of sputter deposited titanium oxide electrodes", tesis para optar el grado de doctor en ciencias, Universidad Nacional de Ingeniría, 2001.

8. Parthiban S, Anuratha KS, Arunprabaharan S, Abinesh S, Lakshminarasimhan N. Enhanced dye-sensitized solar cell performance using TiO2:Nb blocking layer deposited by soft chemical method. Ceramics International. 2015;41(1):205-9.

9. Wei X, Liu J, Liu X-W. Ultrafine dice-like anatase TiO2 for highly efficient dyesensitized solar cells. Solar Energy Materials and Solar Cells. 2015;134:133-9.

10. Liu J, Luo J, Yang W, Wang Y, Zhu L, Xu Y, et al. Synthesis of Single-Crystalline Anatase TiO2 Nanorods With High-Performance Dye-Sensitized Solar Cells. Journal of Materials Science \& Technology. 2015;31(1):106-9.

11. Tang H, Berger H, Schmid PE, Lévy F, Burri G. Photoluminescence in TiO2 anatase single crystals. Solid State Communications. 1993;87(9):847-50.

12. Wang J, Jiang $\mathrm{Y}$, Zhang $\mathrm{Z}$, Zhang $\mathrm{X}$, Ma T, Zhang $\mathrm{G}$, et al. Investigation on the sonocatalytic degradation of acid red $\mathrm{B}$ in the presence of nanometer $\mathrm{TiO} 2$ catalysts and comparison of catalytic activities of anatase and rutile $\mathrm{TiO} 2$ powders. Ultrasonics Sonochemistry. 2007;14(5):545-51.

13. Jang HD, Kim S-K, Kim S-J. Effect of particle size and phase composition of titanium dioxide nanoparticles on the photocatalytic properties. Journal of Nanoparticle Research. 2001;3(2-3):141-7.

14. Kumari J, Kumar D, Mathur A, Naseer A, Kumar RR, Thanjavur Chandrasekaran P, et al. Cytotoxicity of TiO2 nanoparticles towards freshwater sediment microorganisms at low exposure concentrations. Environmental Research. 2014;135:333-45.

15. Dalai S, Pakrashi S, Joyce Nirmala M, Chaudhri A, Chandrasekaran N, Mandal AB, et al. Cytotoxicity of $\mathrm{TiO} 2$ nanoparticles and their detoxification in a freshwater system. Aquatic Toxicology. 2013;138-139:1-11. 
16. Melquiades FL, Ferreira DD, Appoloni CR, Lopes F, Lonni AG, Oliveira FM, et al. Titanium dioxide determination in sunscreen by energy dispersive X-ray fluorescence methodology. Analytica Chimica Acta. 2008;613(2):135-43.

17. Fais LMG, Fernandes-Filho RB, Pereira-da-Silva MA, Vaz LG, Adabo GL. Titanium surface topography after brushing with fluoride and fluoride-free toothpaste simulating 10 years of use. Journal of Dentistry. 2012;40(4):265-75.

18. Su C-Y, Tang H-Z, Chu K, Lin C-K. Cosmetic properties of TiO2/mica-BN composite powder prepared by spray drying. Ceramics International. 2014;40(5):6903-11.

19. Zhao X, Li J, Liu Y, Zhang Y, Qu J, Qi T. Preparation and mechanism of TiO2-coated illite composite pigments. Dyes and Pigments. 2014;108:84-92.

20. Roberto Colonia VM, José Solís, Mónica Gómez. Síntesis de nanopartículas de ZnO2 empleando ultrasonido: caracterización estructural y morfológica para aplicaciones bactericidas. Rev Soc Quím Perú. 2013;79(2): 126 - 135

21. Legrini O, Oliveros E, Braun A. Photochemical processes for water treatment. Chemical reviews. 1993;93(2):671-98.

22. Condon JB. Surface area and porosity determinations by physisorption: measurements and theory: Elsevier; 2006.

23. IUPAC. goldbook.iupac.org.

24. Yu JC, Yu, Ho, Jiang, Zhang. Effects of F- Doping on the Photocatalytic Activity and Microstructures of Nanocrystalline TiO2 Powders. Chemistry of Materials. 2002;14(9):3808-16. 